\title{
The spatial distribution of injuries in need of surgical intervention in Nepal
}

\author{
Shailvi Gupta, ${ }^{1}$ Thomas A. Groen, ${ }^{2}$ Barclay T. Stewart,, ${ }^{3,4}$ Sunil Shrestha, ${ }^{5}$ \\ David A. Spiegel, ${ }^{6}$ Benedict C. Nwomeh, ${ }^{7}$ Reinou S. Groen, ${ }^{8}$ Adam L. Kushner ${ }^{9}$ \\ 1 University of California, San Francisco East Bay and Surgeons OverSeas, Oakland, CA, \\ USA; ${ }^{2}$ University of Twente, Enschede, The Netherlands; ${ }^{3}$ University of Washington, \\ Seattle, WA, USA; ${ }^{4}$ Department of Interdisciplinary Sciences, Stellenbosch University, \\ Cape Town, South Africa; ${ }^{5}$ Nepal Medical College, Kathmandu, Nepal; ${ }^{6}$ Children's Hospital \\ of Pennsylvania, Philadelphia, PA, USA; ${ }^{7}$ Nationwide Children's Hospital and Surgeons \\ OverSeas, Columbus, $\mathrm{OH}$; ${ }^{8} \mathrm{~J}$ ohns Hopkins University School of Medicine and Surgeons \\ OverSeas, Baltimore, MD; 9 Johns Hopkins Bloomberg School of Public Health \\ and Surgeons OverSeas, Baltimore, MD, USA
}

\begin{abstract}
Geographic information system modelling can accurately represent the geospatial distribution of disease burdens to inform health service delivery. Given the dramatic topography of Nepal and a high prevalence of unmet surgical needs, we explored the consequences of topography on the prevalence of surgical conditions. The Nepalese Surgeons OverSeas Assessment of Surgical Need (SOSAS) is a validated, countrywide, cluster randomised survey that assesses surgical need in lowand middle-income countries; it was performed in Nepal in 2014. Data on conditions potentially affected by topography (e.g. fractures, hernias, injuries, burns) were extracted from the database. A national digital elevation model was used to determine altitude, aspect, slope steepness and curvature of the SOSAS survey sites. Forward stepwise linear regression was performed with prevalence of each surgical con-
\end{abstract}

\footnotetext{
Correspondence: Shailvi Gupta, University of California, San Francisco East Bay and Surgeons OverSeas, 1411 East 31st Street, 94602 0akland, CA, USA. Tel: +1.972.841.0242 - Fax: +1.510.437.5127.

E-mail: Shailvi.gupta@gmail.com
}

Key words: Global surgery; Geographic information system; Public health; Topography; Nepal.

Acknowledgments: this study would not have been possible without the gracious help of the Nepali enumerators and field supervisors who graciously donated their time and expertise. Special thanks to Surgeons OverSeas and the Association for Academic Surgery who provided the funding to make this study possible.

Received for publication: 23 March 2015.

Revision received: 24 September 2015.

Accepted for publication: 16 October 2015.

(C) Copyright S. Gupta et al., 2016

Licensee PAGEPress, Italy

Geospatial Health 2016; 11:359

doi:10.4081/gh.2016.359

This article is distributed under the terms of the Creative Commons Attribution Noncommercial License (CC BY-NC 4.0) which permits any noncommercial use, distribution, and reproduction in any medium, provided the original author(s) and source are credited. dition as the response variable and topographic data as explanatory variables. The highest correlation coefficient was for models predicting hernias and fractures, both explaining $21 \%$ of the variance. The model fitted to death due to fall would become significant when an outlier was excluded $\left(\mathrm{P}<0.001 ; \mathrm{R}^{2}=0.27\right)$. Excluding the outlier yielded a better-fitted model to burn injury (stepwise regression) without any explanatory variables. Other models trended towards a correlation, but did not have sufficient power to detect a difference. This study identified slight correlation between elevation and the prevalence of hernias and fall injuries. Further investigation on the effects of topography and geography on surgical conditions is needed to help determine if the data would be useful for directing allocation of surgical resources.

\section{Introduction}

With increasing empirical data globally, surgical care is gaining momentum as a public health priority (Groen et al., 2012; Petroze et al., 2013; Gupta et al., 2015; Bae et al., 2011; Meara et al., 2014). Global estimations conclude that $73.6 \%$ of all surgical operations are carried out in the wealthiest nations, while only $3.5 \%$ of the operations are performed in the world's poorest nations (Weiser et al., 2008). Approximately 2 billion people lack access to an operating room (Funk et al., 2010). Despite the recent interest in global surgical needs, there is still a paucity of objective data needed to define which barriers make access to surgical care so difficult in the world's poorest nations, and if surgical conditions vary depending on geospatial considerations globally. Geographic information systems (GIS) are increasingly used to help model the geospatial distribution of diseases (Rocha et al., 2014; Faierman et al., 2014; Tollefson et al., 2015). Such modelling can provide an accurate, geographic representation of the spatial variation of disease burdens, which can help to guide policymakers in developing future service plans, tackling this aspect of healthcare access. In addition to planning for the location of healthcare services with the aim of providing support where the need is greatest, geospatial modelling can also be used to investigate the extent to which biophysical conditions, such as the presence of parasite reservoirs or how the topography of a country affects the prevalence of medical conditions (Osei et al., 2010). Nepal is a South Asian country with just over 27 million people. Situated between China in the North and India in the South, West and East, Nepal is a landlocked nation with diverse geography, housing eight of the world's ten largest mountains in addition 
to numerous lower hills as well as lakes (World Bank, 2013). The terrain rises from a low of $59 \mathrm{~m}$ above the mean sea level in the Terrai Region at the northern rim of the Gangetic Plain to $8848 \mathrm{~m}$, representing Earth's highest peak Mt. Everest (World Bank, 2013). This wide range of altitudes, and the associated variation in topographic conditions (from gentle flat areas to steep, rugged terrain), provides a useful case study to explore the possible consequences of topography on the prevalence of surgical conditions, such as fractures, hernias and burns. This is an important aspect as was vividly brought home by the devastation of the country in April 2015 through the Gorkha Earthquake, one of the worst natural disasters in its history, which killed more than 8000 people and injuring more than 23,000 (Central News Network, 2015). Such an event in an already low-income and politically fragile country such as Nepal further complicates access to surgical care.

Prior to the 2015 earthquake, the Surgeons OverSeas Assessment of Surgical Need (SOSAS) survey was used in Nepal in 2014 to assess the prevalence of surgical conditions and avoidable deaths. The objective of the present study is to use the data to explore the effect of topography on prevalence of surgical conditions in Nepal.

\section{Materials and Methods}

The SOSAS survey, a validated, countrywide, cluster randomised, cross-sectional survey, was performed in Nepal from May 25 to June 12, 2014. It consists of two parts, the first of which concerns household demographic data, access to healthcare and household member deaths within the past year. The second part randomly selects two household members for a verbal head-to-toe examination focusing on six anatomical regions: i) face, head and neck; ii) chest and breast; iii) abdomen; iv) groin and genitalia; v) back; and vi) extremities. Each respondent verbally elicits symptoms or experiences associated with a general spectrum of surgical conditions, such as wounds, swellings, deformities, burns and injuries. The SOSAS survey has been described in more detail previously (Groen et al., 2012).

\section{Sampling}

A two-stage cluster sampling was performed. First, 15 of the 75 districts proportional to the population were randomly selected, after which 45 Village Development Committees (VDCs) were randomly selected (Figure 1), three for each district after stratification for the urban to rural population distribution (two rural to one urban). This methodology was similar to that used by the Demographic and Health Surveys normally carried out in Nepal (Nepal Ministry of Health and Population, Department of Health Services, 2012). A total sample size of 1350 households was used. The sample size estimation was calculated from a prevalence of unmet surgical needs of $5 \%$, reported by a pilot study of SOSAS in Nepal in January 2014 (Gupta et al., 2014). All surveys were administered in Nepalese and the responses recorded in English via paper forms. Appropriate ethical approval was obtained prior to study execution. Verbal consent was obtained from all respondents prior to survey (parental consent, oral assent and/or parental permission was obtained for individuals younger than 18 years). Individuals noted to be cognitively impaired by household members were excluded from the study.

\section{The Surgeons OverSeas Assessment of Surgical Need database}

By consensus among the authors, surgical conditions that might be affected by altitude were chosen for inclusion in this study. Given the increased strenuous exercise needed at higher altitudes, the augmented risk of falling from heights and lower temperatures (leading to the necessity of fire for warmth), data on fractures, hernias, fall injuries (both fatal and non-fatal), burn injuries, and unmet surgical needs in general were extracted from the SOSAS database and used for the analysis. Unmet surgical need was defined as the affliction of an individual, who had reported a current (within one month) condition that he or she perceived needed at least a surgical consultation, which could not be accessed. Prevalence was calculated as the number of recordings of a medical condition relative to the total number of people surveyed.

The spatial database was constructed as follows. A digital elevation model (DEM) for the whole of Nepal with a resolution of 90 by $90 \mathrm{~m}$ (Figure 2A) was downloaded from the United States Geological Survey (USGS), created by the shuttle radar topography mission (SRTM) (http://srtm.usgs.gov/). Next to altitude, aspect (degrees from north), slope steepness (in degrees) and curvature (the rate at which the slope changes) were calculated from the DEM. For every location where
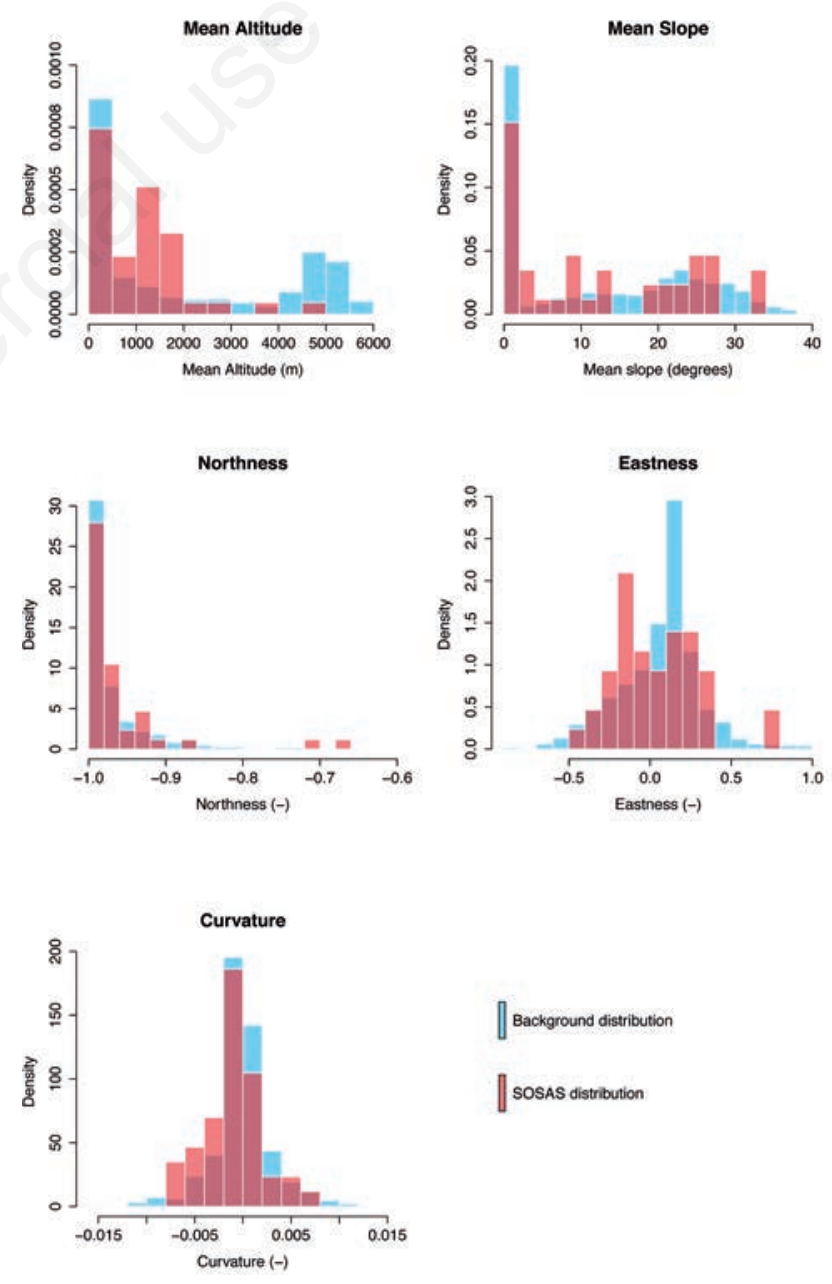

Figure 1. Distribution of topographical conditions that were used in this study from a random selection of 1000 locations in Nepal (i.e. the background) and sites sampled by the Surgeons OverSeas Assessment of Surgical Need (SOSAS). Darker red represents overlapping between background and SOSAS distribution. 
SOSAS data had been collected, average and standard deviation of these factors were calculated within a $5 \mathrm{~km}$ radius from each site. Aspect was then converted to northness and eastness, which converts the degrees from north to a factor with a more straightforward interpretation. Thus, minimum northness (i.e. straight south) becomes -1, while maximum (i.e. straight north) $=+1$. Similarily, west signifies low levels of eastness (i.e. straight west is minimum eastness $=-1$ ), and high values indicate east (i.e. straight east $=+1$ ).

\section{Statistical analyses}

Simple forward stepwise linear regression was performed in $R(R$ Development Core Team, 2014) with prevalence of surgical conditions as response and topographic explanatory variables. Model selection was based on the Akaike Information Criterion (AIC) (Akaike, 1978). The AIC gives a goodness of fit indication based on the number of explanatory variables included and sample size of the dataset. Preference is given to models with fewer explanatory variables. Before fitting the models, collinearity was checked by means of variance inflation factors (VIFs). A VIF upper threshold of 10 was used as criterion to identify too strong collinearity (Quinn and Keough, 2002). After final models were fitted, normality of residuals was checked to ensure that distribution of data was in accordance with model assumptions. Additionally, the degree of representativeness of SOSAS clusters for the topography in the region was made visible by creating histograms of the tested topographic determinants against a background set of 1000 random points in the same area.

\section{Results}

The representativeness of the SOSAS sample for topography is shown in Figure 2B. The SOSAS database covers the distribution of topographic conditions well except for the really high (i.e. $>5000 \mathrm{~m}$ ) and steep (i.e. $>34^{\circ}$ ) areas. Table 1 describes the variables retained by the stepwise procedure and the evidence for association with the selected surgical conditions. Because the variable selection was based on AIC, not every variable necessarily proves significant $(\mathrm{P}<0.05)$. Next to variables selected and their significance, overall model performance is expressed by means of the variance explained $\left(\mathrm{R}^{2}\right)$ and the overall model significance based on an F-test. For a graphical assessment of model fits, observed versus modelled scatter plots are provided in Figure 3. Overall model significance was low. The overall model performance was highest for the model explaining hernias and fractures (both 21\% explained variance). For the models fitted to fall as a cause
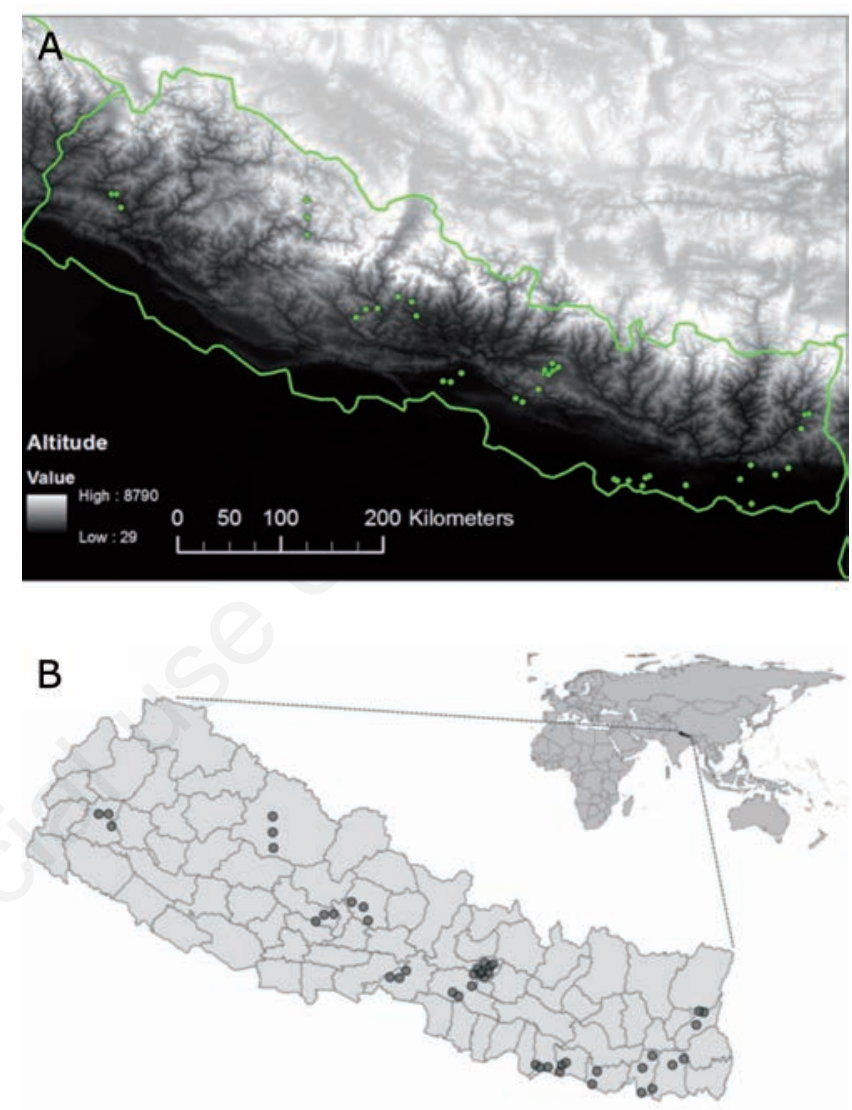

Figure 2. Digital elevation model of Nepal based on United States Geological Survey shuttle radar topography mission data (A) and location of clusters included in the Surgeons OverSeas Assessment of Surgical Need database (B).

Table 1. Model summary for each surgical condition analysed.

\begin{tabular}{|c|c|c|c|c|c|c|}
\hline & Fractures & Hernias & Death due to fall & Fall & Surgical needs & Burning injury \\
\hline Intercept & $<0.001$ & 0.01 & 0.07 & $<0.001$ & $<0.001$ & 0.007 \\
\hline Mean altitude (m asl) & 0.01 & 0.06 & 0.08 & 0.03 & & \\
\hline \multicolumn{7}{|l|}{ Standard altitude (m asl) } \\
\hline Mean slope (degrees) & 0.007 & 0.01 & & 0.11 & & 0.13 \\
\hline Standard slope (degrees) & 0.08 & & & & & \\
\hline \multicolumn{7}{|l|}{ Mean curvature (ratio) } \\
\hline Northness (ratio) & & 0.03 & & & & \\
\hline Eastness (ratio) & 0.18 & & & & & \\
\hline Model R2 & 0.21 & 0.21 & 0.07 & 0.11 & na & 0.05 \\
\hline Overall P & 0.05 & 0.02 & 0.08 & 0.10 & na & 0.13 \\
\hline
\end{tabular}

asl, above the mean sea level; na, not available. Values indicate P values of the partial slopes of every model based on t-tests, except for $\mathrm{R}^{2}$ (variance explained by the model) and the overall $\mathrm{P}$ value, which is based on an F-test (best fit statistical comparison). Italics indicates significance at $\mathrm{P}<0.05$. 
of death (Figure 3C) and burn injury (Figure 3F), one single location had a large leverage on the model. When outliers were excluded, the model fitted to fall injury as a cause of death becomes significant $\left(\mathrm{P}<0.001 ; \mathrm{R}^{2}=0.27\right)$; though excluding the outlier did not make the model fitted to the burn injury model significant.

\section{Discussion}

The concept of access to healthcare has been defined as those dimensions that describe the potential and actual entry of a given population to the health care delivery system. The availability of health care is a component in this concept and refers to the volume and distribution of medical resources in an area (Andersen et al., 1983). Much research on global surgery has equated access of adequate surgical care with the availability of trained surgeons and nurses as well as physical facilities, such as operating theatres, autoclaves, utensils, etc., while other research papers haves focused on characteristics of the population, such as demographics, income, perceived need for surgical care, transport to a primary healthcare facility and severity of symptoms (Groen et al., 2012; Kushner et al., 2010; LeBrun et al., 2014; Ologunde et al., 2014). However, apart from patients, staff, buildings, instruments, affordability and cultural acceptability of care there is another important component: i.e. geographical accessibility, specifically targeted towards areas with particular high needs for care.

Nepal is a country known for a great topographical variation and the SOSAS survey found a 10\% prevalence of unmet surgical needs countrywide, and that was prior to the 2015 earthquake (Gupta et al., 2015). Given the wide range of altitudes and the variation in topographic conditions in Nepal along with a high prevalence of unmet surgical needs, our aim was to explore the possible consequences of topography on the prevalence of surgical conditions. Though not conclusive, our study reveals a correlation (though fairly low) between elevation and the
A

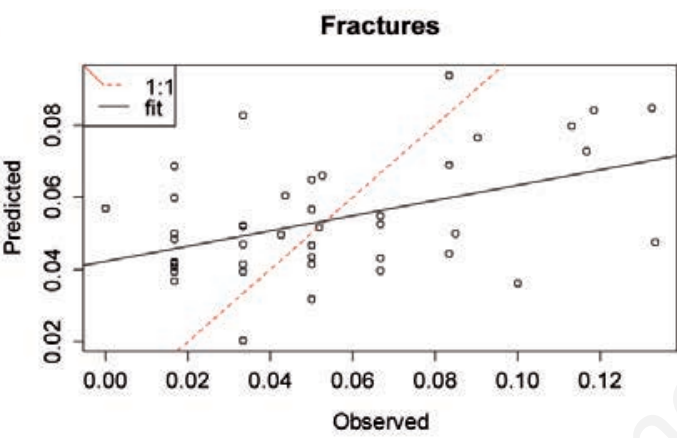

C

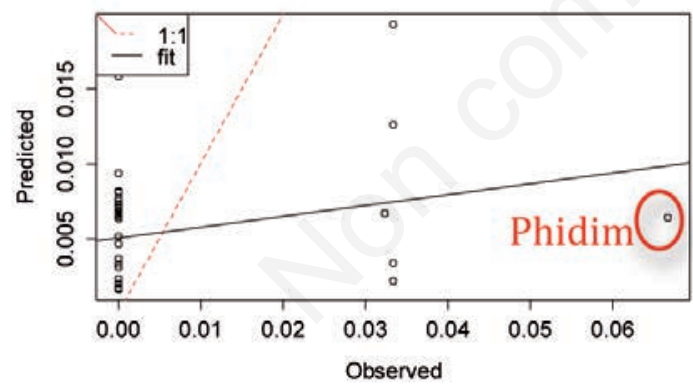

E

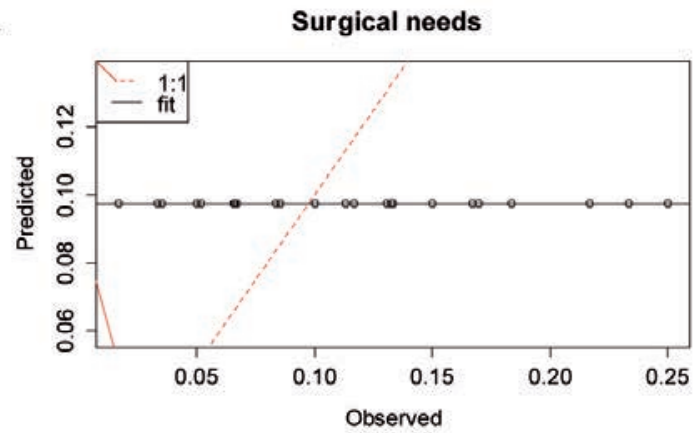

B

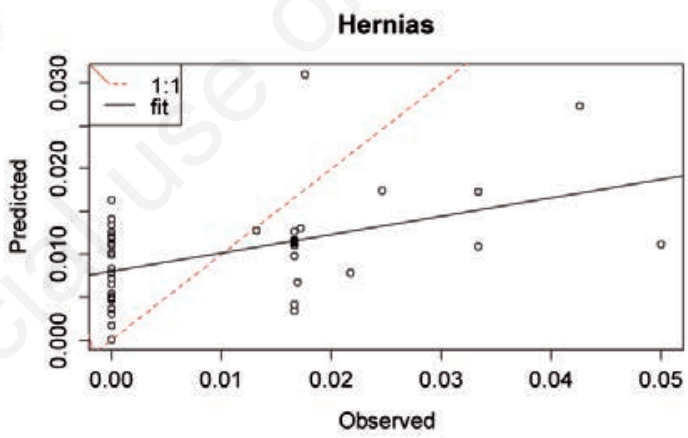

D

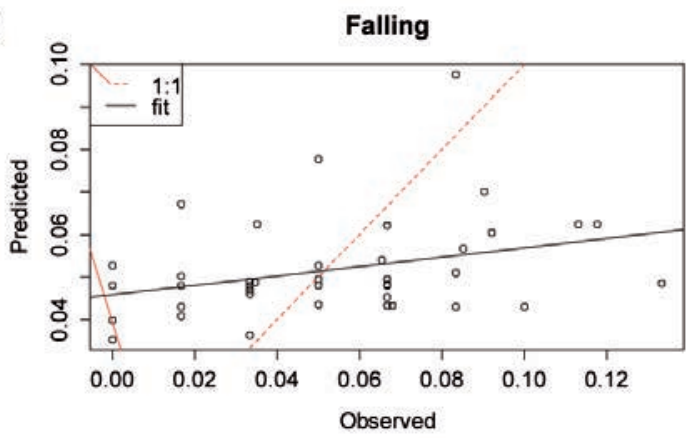

F

Burning injury

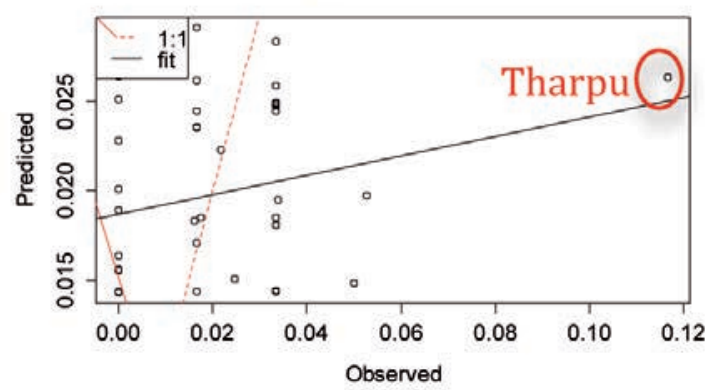

Figure 3. Graphical representations of predicted $v$ s observed prevalence rates of medical conditions as functions of topographical conditions. For C) and F), outliers that had a large leverage on the fitted model are identified. Phidim and Tharpu are Village Development Committees within Nepal that were sampled in this study. 
prevalence of hernias and fall injuries. Geospatial analysis is a useful tool to identify the land cover of particular diseases to: prioritie riskprone areas; examine healthcare misdistribution; and target areas for disease prevention. For example, a study from Bihar state in India mapped out risk prone areas for visceral leishmaniasis or kala-azar sing a GIS approach. This study demonstrated that rural villages surrounded by a higher proportion of transitional swamps and sugarcane plantations had higher sand-fly abundance; and thus, were considered risk-prone areas for man-vector contact (Sudhakar et al., 2006; SalahiMoghaddam et al., 2010). Another study from sub-Saharan Africa used geospatial modelling to optimize rollout plans for anti-retroviral therapy in South Africa (Gerberry et al., 2014). This study found significant geographic variation in the efficiency of interventions in reducing the transmission of the human immunodeficiency virus using geographic targeting to help maximise geographic equity in access to interventions. Such data provide empirical evidence to plan targeted intervention. Similarly, this study sought to identify risk-prone areas for selected conditions requiring surgery, as such information might contribute to the development of targeted interventions to reduce unmet surgical needs in disproportionately affected populations. Furthermore, geospatial analysis can help explore if changes in altitude have a causal association for surgical conditions from a physiological basis. While this study was not equipped to explore a causal relationship between altitude and surgical conditions, previous studies have explored this relationship with regard to infectious diseases, such as pulmonary tuberculosis (Vargas et al., 2004). A Mexican study reveals that altitude had a strong inverse relationship to pulmonary tuberculosis, perhaps related to the well-known changes in alveolar oxygen pressure at different altitudes. Such techniques can help explore if certain surgical conditions are more likely at higher altitudes from a physiological perspective. Our results show that in Nepal, slope correlated with the numbers of both fractures and hernia prevalence, while altitude was correlated with fractures and death due to fall injuries, though not necessarily all fall injuries (non-fatal included). Other data analysed trended toward a correlation, but this study, as an exploratory analysis, did not have sufficient statistical power to detect a difference with respect to other conditions. The correlations noted may be explained, in part, by: i) physiology of living at higher elevations; ii) a greater relative risk for incurring surgical conditions that populations living in more complex terrain have (e.g. falling leading to fracture; repeated strenuous activity leading to hernia); or iii) having less access to appropriate surgical care. However, we did not find evidence for a difference between the prevalence of any unmet surgical need at differing elevations or terrain ruggedness.

Clearly, the large effect that a few observations had on the overall performance of some of the models (Phidim and Tharpu) shows that he spatial spread of these observations and the total sample size was probably not optimal for this study. Nevertheless, these data demonstrate that there may be some relationships between terrain and surgical conditions that need to be better defined. This calls for efforts to collate databases, such as the SOSAS surveys, to analyse these relationships, which then in turn can be used to prioritse the allocation of scarce surgical resources to areas where they are most needed. As geospatial technology continues to improve, such technologies should be included in current data collection strategies and evaluations.

Our study was executed prior to the Gorkha earthquake in 2015, thus many more individuals in mountainous regions without access to healthcare likely exist than were noted in our study. In order to confront this issue, Nepal instituted emergency mobile health clinics postdisaster (World Health Organization, 2015), thus allowing more Nepalese to have access to healthcare. This study has several limita- tions. Limitations inherent to all cross sectional studies with similar random sampling methodology should be considered. Despite the use of data from a robustly sample-sized survey, resources only allowed for 15 of Nepal's 75 districts to be selected. Because these districts were selected proportional to population, this means that more densely populated areas had a higher chance of being selected resulting in less developed regions and less densely populated areas of Nepal being underrepresented. These more rural areas in Nepal tend to be more mountainous and with higher elevations. Thus, the SOSAS study is a good start to explore such topographical relationships, but it is likely that a dedicated study focusing on both the higher and lower elevations in Nepal is needed to build more conclusive results. SOSAS relies on verbally self-reported data, which may be prone to recall bias. However, as part of SOSAS Nepal, a visual physical examination was performed, which validated these verbal reports (Gupta et al., 2015). Despite those limitations, the present results provide a valuable starting point for future studies to evaluate the effect of topographical difference in the prevalence of surgical conditions.

\section{Conclusions}

Given the wide range of altitudes and the variation in topographic conditions in Nepal, we explored the possible consequences of topography on the prevalence of surgical conditions, such as fractures, hernias and burns. Though not conclusive, the study reveals a correlation between elevation and the prevalence of hernias and fall injuries. Further investigation on the effects of topography and geography on surgical conditions is needed to help direct the allocation of resources for surgical care.

\section{References}

Akaike H, 1978. A Bayesian analysis of the minimum AIC procedure. Ann I Stat Math 30:9-14.

Andersen RM, McCutcheon A, Aday LA, Chiu GY, Bell R, 1983. Exploring dimensions of access to medical care. Health Serv Res 18:49-74.

Bae JY, Groen RS, Kushner AL, 2011. Surgery as a public health intervention: common misconceptions versus the truth. B World Health Organ 89:394.

Central News Network, 2015. Death toll in Nepal earthquake tops 8,000. Available from: http://www.cnn.com/2015/05/10/asia/nepal-earthquake-death-toll/

Faierman ML, Anderson JE, Assane A, Bendix P, Vaz F, Rose JA, Funzamo C, Bickler SW, Noormahomed EV, 2014. Surgical patients travel longer distances than non-surgical patients to receive care at a rural hospital in Mozambique. Hum Res Dev 7:60-6.

Funk LM, Weiser TG, Berry WR, Lipsitz SR, Merry AF, Enright AC, Wilson IH, Dziekan G, Gawande AA, 2010. Global operating theatre distribution and pulse oximetry supply: an estimation from reported data. Lancet 376:1055-61.

Gerberry DJ, Wagner BG, Garcia-Lerma JG, Heneine W, Blower S, 2014. Using geospatial modeling to optimize the rollout of antiretroviralbased pre-exposure HIV interventions in sub-Saharan Africa. Nat Commun 5:5454.

Groen RS, Samai M, Petroze RT, Kamara TB, Yambasu SE, Calland JF, Kingham TP, Guterbock TM, Choo B, Kushner AL, 2012. Pilot testing of a population-based surgical survey tool in Sierra Leone. 
World J Surg 36:771-4.

Groen RS, Samai M, Stewart K, Cassidy LD, Kamara TB, Yambasu SE, Kingham TP, Kushner AL, 2012. Untreated surgical conditions in Sierra Leone: a cluster randomised cross-sectional, countrywide survey. Lancet 380:1082-7.

Gupta S, Ranjit A, Shrestha R, Wong EG, Robinson WC, Shrestha S, Nwomeh BC, Groen RS, Kushner AL, 2014. Surgical needs of Nepal: pilot study of a population based survey in Pokhara, Nepal. World J Surg 38:3041-6.

Gupta S, Shrestha S, Ranjit A, Nagarajan N, Groen RS, Kushner AL, Nwomeh BC, 2015. Surgical care in Nepal: conditions, preventable deaths, procedures and validation of a countrywide survey. Brit $\mathrm{J}$ Surg 102:700-7.

Kushner AL, Cherian MN, Noel L, Spiegel DA, Groth S, Etienne C, 2010. Addressing the Millennium Development Goals from a surgical perspective: essential surgery and anesthesia in 8 low-and middleincome countries. Arch Surg 145:154-9.

LeBrun DG, Chackungal S, Chao TE, Knowlton LM, Linden AF, Notrica MR, Solis CV, McQueen KA, 2014. Prioritizing essential surgery and safe anesthesia for the Post-2015 Development Agenda: operative capacities of 78 district hospitals in 7 low- and middle-income countries. Surgery 155:365-73.

Meara JG, Hagander L, Leather AJM, 2014. Surgery and global health: a Lancet Commission. Lancet 383:9911.

Nepal Ministry of Health and Population, Department of Health Services, 2012. Annual report. Available from: dohs.gov.np/wpcontent/uploads/2014/04/Annual_Report_2068_69.pdf

Ologunde R, Maruthappu M, Shanmugarajah K, Shalhoub J, 2014. Surgical care in low and middle-income countries: burdens and barriers. Int J Surg 12:858-63.

Osei FB, Duker AA, Augustijn PWM, Stein A, 2010. Spatial dependency of cholera prevalence on potential cholera reservoirs in an urban area, Kumasi, Ghana. Int J Appl Earth Obs 12:331-9.
Petroze R, Groen RS, Niyonkuru F, Mallory M, Ntaganda E, Joharifard S, Guterbock T, Kushner AL, Kyamanywa P, Calland FJ, 2013. Estimating operative disease prevalence in a low income country: results of a nationwide population survey in Rwanda. Surgery 153:457-64.

Quinn GP, Keough MJ, 2002. Experimental design and data analysis for biologists. Cambridge University Press, Cambridge, UK.

R Development Core Team, 2014. R: a language and environment for statistical computing. R Foundation for Statistical Computing, Vienna, Austria. Available from: http://www.R-project.org/

Rocha CM, Kruger E, Whyman R, Tennant M, 2014. Predicting geographically distributed adult dental decay in the greater Auckland region of New Zealand. Community Dent Hlth 31:85-90.

Salahi-Moghaddam A, Mohebali M, Moshfae A, Habibi M, Zarei Z, 2010. Ecological study and risk mapping of visceral leishmaniasis in an endemic area of Iran based on a geographical information systems approach. Geospat Health 5:71-7.

Sudhakar S, Srinivas T, Palit A, Kar SK, Battacharya SK, 2006. Mapping of risk prone areas of kala-aza (visceral leishmaniasis) in parts of Bihar State, India: an RS and GIS approach. J Vector Dis 43:115-22.

Tollefson TT, Shaye D, Durbin-Johnson B, Mehdezadeh 0, Mahomva L, Chidzonga M, 2015. Cleft lip palate in Zimbabwe: estimating the distribution of surgical burden of disease using GIS. Laryngoscope 125(Suppl.1):1-14.

Vargas MH, Furuya MEY, Perez-Guzman C, 2004. Effect of altitude on the frequency of pulmonary tuberculosis. Int $\mathrm{J}$ Tuberc Lung $\mathrm{D}$ 8:1321-4.

Weiser TG, Regenbogen SE, Thompson KD, Haynes AB, Lipsitz SR, Berry WR, Gawande AA, 2008. An estimation of the global volume of surgery: a modeling strategy based on available data. Lancet 372:139-44.

World Bank, 2013. Nepal country at a glance. Available from: http://www.worldbank.org/en/country/nepal 